DAN ZAHAVI

\title{
Der Sinn der Phänomenologie: Eine methodologische Reflexion
}

Eine der hier zu behandelnden Fragen betrifft die Rolle, die das Bewusstsein bei der Erzeugung und Bildung von Sinn spielt. Eine der gängigen Einschätzungen der Entwicklung der nach-Husserl'schen Phänomenologie charakterisiert diese durch die zunehmende Erkenntnis, die Erzeugung von Sinn werde nicht von der konstitutiven Leistung des intentionalen Bewusstseins beherrscht. Da dies nun aber der ursprüngliche Husserl'sche Anspruch gewesen sein soll, kann daraus eben nur die Schlussfolgerung gezogen werden, dass wir die Beschränktheiten der Husserl'schen Phänomenologie eingestehen müssen, und dass die Phänomenologen von heute - so sie überhaupt noch an einer Klärung der Frage der Sinnbildung interessiert sind - anderen als den von Husserl in seinen klassischen Werken meisterlich vorgezeichneten Bahnen folgen müssen.

Die Einladung zu einem Beitrag zum Thema „Phänomenologie der Sinnereignisse" stellte mich vor zwei Herauforderungen: zum einen zu prüfen, ob die Husserl'sche Phänomonologie nicht doch Ressourcen von bleibendem Wert enthält - ich verstehe insofern meine Aufgabe als eine defensio Husserls -, zum anderen, meine Überlegungen möglichst mit der Arbeit der empirischen Psychologie in Verbindung zu bringen.

Ich beginne mit der interdisziplinären Fragestellung. Aus vielerlei Gründen ist es für Phänomenologen wichtig, sich mit der empirischen Forschung vertraut zu machen. So sind die Ergebnisse von Psychopathologie, Entwicklungspsychologie und Kognitiver Psychologie nicht nur für ein phänomenologisches Verständnis etwa von Intersubjektivität, Selbsterfahrung oder Gedächtnis relevant; die Auseinandersetzung mit derartiger Forschung vermag überdies unser eigenes Verständnis vom einzigartigen Beitrag der Phänomenologie zu schärfen. Um es anders und ein wenig kritischer zu sagen, bereits ein kurzer Blick auf einige Ergebnisse der Kognitiven Psychologie lehrt uns meines Erachtens, dass der eingangs bezeichnete Ansatz der zeitgenössischen Phänomenologie uns irgendwie auf Abwege führt: Dass eines der Hauptunterscheidungsmerkmale der zeitgenössischen Phänomenologie die Einsicht gewesen sein soll, die Erzeugung von Sinn sei nicht vom intentionalen Bewusstsein be- 
herrscht, kann schlichtweg nicht stimmen. Und zwar, weil diese Behauptung weder neu noch spezifisch phänomenologisch, sondern im Gegenteil eine seit langem von einer Vielzahl von Theoretikern und auch empirischen Forschern vorgetragene These ist.

\section{Nicht-bewusste Informationsverarbeitung}

Zur Veranschaulichung möchte ich kurz einige Ergebnisse der Kognitiven Psychologie durchgehen. Ein häufig verwendetes Musterbeispiel ist als das Zweikanal-Experiment bekannt. Versuchspersonen werden per Kopfhörer jeweils über das rechte und das linke Ohr gleichzeitig zwei Informationsströmen ausgesetzt; sie werden dann aufgefordert, nur auf einen der beiden Informationsströme zu achten und ihm zu folgen, das heißt all das laut zu wiederholen, was sie auf diesem einen Ohr hören. Dies verlangt den Versuchspersonen eine Aufmerksamkeit in solch hohem Maße ab, dass sie offensichtlich nicht zur Kenntnis nehmen können, was ihnen auf dem anderen Ohr vorgetragen wird. Sie sind somit außerstande, irgendetwas von dem, was auf diesem anderen Ohr gesagt wurde, zu identifizieren, wiederzugeben oder sich in Erinnerung zu rufen. Und doch scheint aus verschiedenen Untersuchungsergebnissen hervorzugehen, dass selbst, wenn es an jeglicher bewusster Aufmerksamkeit für diese unbeachteten Stimuli fehlt, deren Sinn den Hörer berühren und sogar seine Interpretation der beachteten Botschaften beeinflussen kann. So führten beispielsweise in einem Experiment, das von seiner Beschreibung her einen ziemlich barbarischen Eindruck macht, Wörter, die zuvor mit Stromstößen verknüpft waren, auch dann weiterhin zu Veränderungen im Hautleitwiderstand, wenn sie dem nicht achthabenden Ohr präsentiert wurden. ${ }^{1}$ Dies geschah sogar bei Wörtern, die semantisch in einem Bezug zu den konditionierten Wörtern stehen. ${ }^{2}$

Weitere empirische Resultate haben gewisse philosophische Platitüden, die sich auf die Rolle beziehen, die die bewusste Erfahrung bei der Kontrolle intentionaler Handlungen spielt, in Frage gestellt. Bei der berühmten Ebbinghaus-Illusion bekommt man zwei Zielscheiben derselben Größe vorgelegt.

1 B. J. Baars, In the Theater of Consciousness. The Workspace of the Mind, Oxford: Oxford University Press 1997, S. 17.

2 M. Velmans, Understanding Consciousness, London: Routledge 2000, S. 199. 


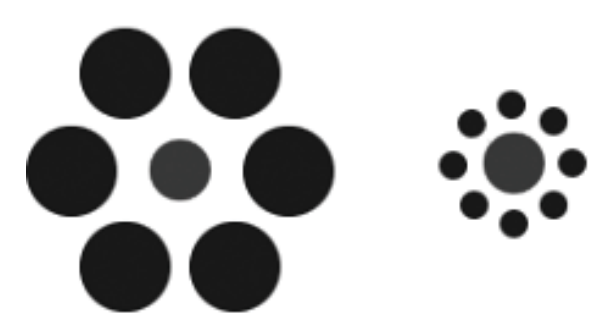

Die eine ist von einem Kreis aus Scheiben umgeben, von denen jede größer ist als die Zielscheibe. Die andere ist von einem Kreis aus Scheiben umgeben, von denen jede kleiner ist als die Zielscheibe. Die von dem Kreis aus größeren Scheiben umgebene Zielscheibe wird normalerweise als kleiner erlebt als die von einem Kreis kleinerer Scheiben umgebene Zielscheibe. Diese Wahrnehmungsillusion ist recht stabil; doch wenn Sie dann anschließend aufgefordert werden, eine der Zielscheiben aufzunehmen, werden Sie den Umfang des Zugriffs ihrer Hand an der tatsächlichen Größe der Scheiben bemessen. Das heißt, Ihre Griffweite wird trotz der Tatsache, dass die Scheiben von Ihnen als von unterschiedlicher Größe erlebt werden, gleich groß sein. Das brachte manche Forscher zu der These, es gäbe zwei Wahrnehmungssysteme. Das eine ist in die Handlungssteuerung, das andere in die Erzeugung bewusster Erfahrung involviert, und so gelangte man zu der Ansicht, dass Handlungsparameter durch nicht-bewusste Informationsverarbeitungsmechanismen auf einer niedrigen Stufe bestimmt sind, die sich über den Inhalt Ihres bewussten Erlebens einfach hinwegsetzen. ${ }^{3}$

Nehmen wir an, ein taktiler Reiz werde auf die Haut ausgeübt, und die Versuchsperson soll einen Knopf drücken, sobald sie ihn verspürt. Es braucht nur wenige Millisekunden, bis dass der Reiz auf der Haut die Oberfläche des Kortex erreicht, und es braucht nur ca. 100 Millisekunden, um auf den Reiz zu reagieren. Doch gewissen Untersuchungsergebnissen zufolge braucht es ca. 500 Millisekunden, bis dass ein Bewusstsein des Reizes entsteht. Nichtsdestotrotz haben wir den subjektiven Eindruck, dass wir reagieren, nachdem wir fühlen, dass etwas die Haut berührt. Doch das ist erklärtermaßen eine bloße Illusion, das Ergebnis einer Post-hoc-Produktion des Gehirns. ${ }^{4}$

3 J. Campbell, „The Role of Demonstratives in Action-Explanation“, in: J. Roessler, N. Eilan (Hg.), Agency and Self-awareness, Oxford: Clarendon Press 2003, S. 150-164, hier: S. 157-158.

$4 \mathrm{M}$. Velmans, „Is Human Information Processing Conscious?“, in: Behavioral and Brain Sciences 14 (1991), S. 651-726 [hier zitiert nach http://cogprints.org/593/00/199802003.html]. 
In einem Artikel von 1991 wertete der britische Psychologe Max Velmans eine große Menge von Daten aus, die mit der Frage zu tun haben, wie das Bewusstsein in die menschliche Informationsverarbeitung eingreift. Er kommt zu folgendem Resümee:

„In the above examples, we have ranged over all the main phases of human information processing - from information encoding, storage, retrieval, and transformation to output. We have considered the role of consciousness in the analysis and selection of stimuli, in learning and memory, and in the production of voluntary responses, including those requiring planning and creativity ... [T] here is a sense in which the execution of none of these tasks is ,conscious' ... Rather, one becomes aware of a stimulus only after one has analyzed and selected it, and aware of one's own response only after one has executed it. This applies not only to simple, automatic responses (such as pressing a button or detecting a stimulus) but also to complex, novel, voluntary responses. "5

Bewusstsein scheint demzufolge eher eine Form von Output als etwas zu sein, das direkt in die kognitive Verarbeitung involviert ist, und gemäß Velmans Schlussfolgerung ,this appears to support epiphenomenalism “. ${ }^{6}$ Demnach sollte nach Ansicht mancher bewusste Erfahrung dieselbe Beziehung zum Gesamtorganismus haben wie die Dampfpfeife zur Maschine der Lokomotive. Kurz gesagt, vielen, wenn nicht den meisten Kognitionswissenschaftlern zufolge ist das Ich gewiss nicht Herr im eigenen Haus. Weder leitet es Handlungen noch kontrolliert es die Sinnerzeugung. Letztere scheint unabhängig von unserem Bewusstsein, unserer Kontrolle und Anstrengung zustande zu kommen.

Einige dieser Schlussfolgerungen sind sicherlich anfechtbar. Doch geht es mir im Weiteren nicht um eine Korrektur oder Kritik dieser Ergebnisse; vielmehr sollen sie uns zu einer Neubetrachtung des spezifischen Beitrags der Phänomenologie verhelfen. Wenn die Ansprüche der Phänomenologie von ganz anderer Art sein sollen als die gerade vorgetragenen und ich gehe davon aus, dass das die mehrheitliche Einstellung der Phänomenologen ist -, dann müssen wir uns fragen: Warum ist das so?

\section{Die Reduktion}

Aus einer husserlianischen Perspektive ist die Antwort ziemlich eindeutig, denn Husserl hat immer wieder darauf bestanden, dass wir keine Aussicht haben zu verstehen, worum es der Phänomenologie geht, wenn wir

5 Ebd., S. 24-25.

6 Ebd., S. 26. Es ist allerdings festzuhalten, dass Velmans selbst kein Epiphänomenalist ist. 
die Epoché und die transzendentale Reduktion als irrelevante Spezialprobleme betrachten. ${ }^{7}$ Somit gewährleistet für Husserl gerade der Vollzug der Reduktion den radikalen Unterschied zwischen der phänomenologischen Reflexion und allen anderen Denkweisen. Daher könnte es sich vielleicht lohnen, den folgenden Vorschlag in Betracht zu ziehen: Auf die Frage, in welchem Maße Husserls Phänomenologie gewisse Ressourcen enthält, die für die gegenwärtigen phänomenologischen Untersuchungen der Sinnbildung von Relevanz bleiben, gibt es nur die eine Antwort, dass Husserl eine Reihe methodologischer Erfordernisse aufgelistet hat, die selbst noch heute für jede sich als phänomenologisch verstehende Untersuchung unabdingbar bleiben.

Dieser Vorschlag ist natürlich nicht unumstritten und trifft gewöhnlich auf zwei Arten von Einwänden. Der erste Einwand ist, dass die Reduktion kaum als notwendiges und hinreichendes Merkmal der Phänomenologie zählen kann, da die meisten nach-Husserl'schen Phänomenologen sich übereinstimmend für ihre Verabschiedung aussprechen. Der zweite Einwand ist, dass unabhängig von dem Wert, den der Begriff Reduktion haben mag, dieser sich am Ende doch als unzureichend erweist, weshalb denn auch nachfolgende Phänomenologen sich auf verschiedene Weise um seine Radikalisierung bemüht haben. Ich werde mich im Folgenden mit beiden Einwänden auseinandersetzen.

\section{a. Die internalistische Fehldeutung}

Es ist verschiedentlich behauptet worden, Husserl befürworte einen transzendentalen Idealismus, der sämtliche die äußere Wirklichkeit betreffenden Fragen ausklammert, um sich auf die inneren Strukturen der Erfahrung zu konzentrieren. ${ }^{8}$ Andere haben die These vertreten, Husserls transzendentale Methodologie lege ihn auf eine Form von methodologischem Solipsismus fest, und somit werde fortan die Welt zugunsten der mentalen Repräsentationen ignoriert, welche die Intentionalität ermöglichen.' Weiter wurde behauptet, Husserl habe sämtliche das Sein der

7 E. Husserl, Ideen zu einer reinen Phänomenologie und phänomenologischen Philosophie. Drittes Buch: Die Phänomenologie und die Fundamente der Wissenschaften [Husserliana, Bd. V], hg. von M. Biemel, Den Haag: M. Nijhoff 1971, S. 155; ders., Ideen zu einer reinen Phänomenologie und phänomenologischen Philosophie. Erstes Buch: Allgemeine Einführung in die reine Phänomenologie, S. 200.

8 S. Blackburn, The Oxford Dictionary of Philosophy, Oxford: Oxford University Press 1994, S. 181; D.W. Smith \& R. McIntyre, Husserl and Intentionality, Dordrecht: D. Reidel 1982, Kap. XIV, S. 87 f.

9 H. L. Dreyfus, Being-in-the-World, Cambridge, Mass.: MIT Press 1991, S. 50. 
Wirklichkeit betreffenden Fragen außer Kraft gesetzt und auch der Frage, ob das, worauf wir intentional gerichtet sind, existiert oder nicht, keinerlei Aufmerksamkeit gewidmet. Vor diesem Hintergrund ist es relativ einfach, Husserl zu kritisieren. Man kann so ohne Weiteres einfach behaupten, Husserl habe an der klassischen Subjekt-Objekt-Spaltung festgehalten, was ihn in die Konfrontation mit unausweichlichen Problemen führt. Durch ein methodologisches Vorgehen, das den Geist von der Welt trennt, verliere er nicht nur die Welt aus dem Blick, sondern bleibe auch außerstande, wieder Zugang zu ihr zu erhalten. Aus ähnlichen Gründen büße er auch die Fähigkeit ein, Intersubjektivität und Verleiblichung auf befriedigende Weise darzustellen. Deshalb müsse, so wird von manchen argumentiert, Husserls transzendentale Phänomenologie letztlich zu Gunsten von Heideggers und Merleau-Pontys existenzialer und hermeneutischer Phänomenologie aufgegeben werden.

Meiner Ansicht nach ist diese Kritik von Grund auf fehlgeleitet. Sie ignoriert sämtliche Stellen, an denen Husserl ausdrücklich bestreitet, das wahre Anliegen von Epoché und Reduktion sei die Bezweiflung, Vernachlässigung, Aufgabe oder Ausschließung der Wirklichkeit im Hinblick auf unsere Forschung, und es stattdessen vielmehr als ihr Ziel herausstellt, eine gewisse dogmatische Einstellung gegenüber der Wirklichkeit außer Kraft zu setzen oder zu neutralisieren, was es uns erlaubt, uns enger und direkter auf die Wirklichkeit zu richten, so wie sie gegeben ist. Mit anderen Worten, Epoché und Reduktion implizieren keine ausschließliche Hinwendung an die Innerlichkeit. Sie erlauben uns im Gegenteil, Wirklichkeit auf eine neue Weise, nämlich in ihrer Bedeutung und Bezeigung für das Bewusstsein, zu untersuchen. Kurz gesagt, die Epoché hat einen Einstellungswechsel gegenüber der Wirklichkeit und nicht keinen Ausschluss der Wirklichkeit zur Folge.

Wie Husserl in dem 1931 in Frankfurt, Berlin und Halle gehaltenen Vortrag „Phänomenologie und Anthropologie“ darlegt, wird als Ergebnis der Epoché einzig eine gewisse Naivität ausgeschlossen, und zwar die Naivität, die schlichtweg die Welt für vorgegeben hält und dabei den Beitrag des Bewusstseins ignoriert. ${ }^{10}$ Wiederholt besteht er in diesem Vortrag von 1931 darauf, dass die Wende von einer naiven Erforschung der Welt zu einer reflexiven Erforschung des Bewusstseinsfeldes keine Abwendung von der Welt nach sich zieht, sondern eine Wende, die erstmals ein wahrhaft radikales Erforschen und Verstehen der Welt er-

10 Vgl. E. Husserl, Aufsätze und Vorträge (1922-1937) [Husserliana, Bd. XXVII], hg. von T. Nenon und H. R. Sepp, Den Haag und Dordrecht: Kluwer Academic Publishers 1989, S. 173. 
möglicht. ${ }^{11}$ Auch wenn sich diese reflexive Untersuchung von einer direkten Untersuchung der Welt unterscheidet, bleibt sie doch eine Untersuchung der Wirklichkeit; sie ist nicht die Untersuchung eines andersweltlichen mentalen Reiches. Einzig eine fälschliche Ansicht der Natur von Sinn und Erscheinung würde zu einem solchen Missverständnis führen. So hat Husserl in vielen seiner Schriften immer wieder hervorgehoben, dass der Vollzug von Epoché und Reduktion eine entscheidende Entdeckung möglich mache und infolgedessen als eine Erweiterung (und nicht als eine Beschränkung) unseres Forschungsfeldes anzusehen sei. ${ }^{12}$ Deshalb auch kann Husserl in der Krisis die Durchführung der Epoché mit dem Übergang von einem zweidimensionalen zu einem dreidimensionalen Leben vergleichen. ${ }^{13}$ In Wirklichkeit sei es, wie er in Erste Philosophie II darlegt, tatsächlich besser, den Ausdruck „Ausschaltung“ überhaupt zu meiden, da der Gebrauch dieses Ausdrucks leicht zu der fälschlichen Ansicht führen könne, dass das Sein der Welt kein phänomenologisches Thema mehr sei, obwohl doch die transzendentale Forschung "auch die Welt selbst, nach all ihrem wahren Sein" einschließt. ${ }^{14}$

Diese allgemeine Interpretation lässt sich durch weiteres Material stützen, das sich in dem jüngst veröffentlichten Band 34 der Husserliana, Zur phänomenologischen Reduktion: Texte aus dem Nachlass findet. In diesen zwischen 1926 und 1935 geschriebenen Texten legt Husserl erstmals dar, dass, wenn es heißt, die natürliche Welt sei „ausgeschaltet", dies einfach nur bedeutet, dass der Transzendentalphilosoph aufhören muss, naiv die Welt zu setzen. ${ }^{15}$ Es impliziert nicht, dass ich nicht weiterhin die Welt beobachten und thematisieren und über sie Urteile fällen kann; ich muss dies bloß in einer reflexiven Weise tun, die die Welt als intentionales Korrelat betrachtet. ${ }^{16}$ Anders gesagt, der Vollzug von Epoché und Reduktion bewirkt eine "thematische Umstellung ${ }^{\text {“. }}{ }^{17}$ Fortan wird die Welt

11 Ebd., S. 178.

12 E. Husserl, Cartesianische Meditationen und Pariser Vorträge, S. 66; ders., Die Krisis der europäischen Wissenschaften und die transzendentale Phänomenologie, S. 154.

13 E. Husserl, Die Krisis der europäischen Wissenschaften und die transzendentale Phänomenologie, S. $121 \mathrm{f}$.

14 E. Husserl, Erste Philosophie (1923/24). Zweiter Teil, Theorie der phänomenologischen Reduktion, S. 432.

15 E. Husserl, Zur phänomenologischen Reduktion. Texte aus dem Nachlass (1926-1935) [Husserliana, Bd. XXXIV], hg. von S. Luft, Dordrecht: Kluwer Academic Publishers 2002, S. 21.

16 Ebd., S. 58.

17 Ebd., S. 204. 
als ein Phänomen offenbart, und eben als solche bleibt sie genau im Fokus meiner phänomenologischen Forschung. Um Husserl zu zitieren: ${ }^{18}$

„Welt als ,Phänomen', als Welt in der Epoché, ist doch nur ein Modus, in dem dasselbe Ich, das Welt vorgegeben hat, sich auf diese Vorgegebenheit und was in ihr liegt, besinnt und nicht etwa darum sie und ihre Geltung preisgegeben oder gar einfach zum Verschwinden gebracht hat. "19

Husserl besteht wiederholt darauf, dass die Reduktion als ein ausgesprochener "Sprung der Reflexion“20 angesehen werden muss, der unser Verständnis erweitert, indem er uns einzigartige Einsichten ermöglicht. Zum einen ist die Betrachtung der Welt als ein Phänomen keine Abstraktion, sondern vielmehr die Thematisierung einer ansonsten anonym fungierenden Dimension, nämlich der eigentlichen Dimension der Gegebenheit. Somit bedeutet der Vollzug der Reduktion nach Husserls eigener Formulierung die Befreiung der Welt von einer verborgenen Abstraktion und ihre Offenlegung in ihrer Konkretion als konstituiertes Sinngebilde. ${ }^{21}$ Zugleich befreit die Reduktion auch das Ich von den Beschränkungen seines natürlichen Seins. Mein gewöhnliches natürliches Leben ist Husserl zufolge ein Leben in Selbstentfremdung, da es ein Leben ist, das nichts von seiner Transzendentalität weiß. Die Durchführung der Reduktion entfernt die Scheuklappen, die gewöhnlich den vollen und konkreten transzendentalen Charakter des Lebens verbergen, ${ }^{22}$ sie hebt die Selbstentfremdung auf und hebt die Subjektivität auf eine neue Ebene des transzendentalen Selbstbewusstseins. ${ }^{23}$ Husserl macht geltend, dass damit die Seinsgeltung der Welt weder aufgehoben noch außer Kraft gesetzt wird, sondern dass dies lediglich ihre Relativierung bedeutet. ${ }^{24}$ Folglich beruht die häufig wiederholte Behauptung, die Husserl'sche Reduktion schließe eine Suspendierung von Seinssetzungen ein, schlicht und einfach auf einer Fehldeutung. Wie Husserl es in einem Text aus dem Jahre 1930 darlegt:

„Hat die transzendentale Phänomenologie [...] ihr Werk getan, wenigstens im Allgemeinsten die universale Seins- und Weltstruktur umgriffen, dann ist auch der Sinn der Methode der Epoché, die zu Anfang in ihrer Tragweite unverständlich sein musste, begriffen. Sie leitet zum für mich ersten Absoluten, zu mir als transzendentalem Ich, und indem sie fortleitet zur

18 Ebd., S. 323.

19 Ebd., S. 223, siehe auch S. 83 f.

20 Ebd., S. 219

21 Ebd., S. 225.

22 Ebd., S. 226, S. 233.

23 Ebd., S. 399.

24 Ebd., S. 233. 
Auslegung der Konkretion dieses Ich in der Primordialität und zur Aufklärung der Konstitution der Anderen als anderer Menschen und dann meiner selbst als Mensch in der Welt - die dabei selbst ihren transzendentalen Sinn erhält -, wird die Enthaltung von der Setzung der Welt systematisch aufgehoben. Systematisch werden die transzendentalen Voraussetzungen für sie enthüllt, und als transzendental gültige enthüllt in transzendentaler Evidenz. Und schließlich erwächst die seiende Welt in Seinsgeltung, in derselben, die sie in der Naivität hat, nur jetzt unter Enthüllung der transzendentalen Voraussetzungshorizonte. ${ }^{\text {"25 }}$

\section{b. Die Heidegger'sche Fehldeutung}

Um besser zu begreifen, worauf genau die Reduktion hinausläuft, gehen wir nun zu dem zweiten, dem anspruchsvolleren Einwand gegen die Reduktion weiter. Diesem zufolge liegt das Problem nicht im Begriff der Reduktion per se, sondern darin, dass dieser von Husserl nicht auf eine hinreichend radikale Weise durchdacht worden sei. Als Erster hat vermutlich Heidegger diesen Einwand in der Vorlesung Prolegomena zur Geschichte des Zeitbegriffs vorgebracht, und er ist seitdem von Heideggerianern häufig wiederholt worden. Im Folgenden werde ich mich hauptsächlich auf die von Marion in Réduction et donation vorgelegte Variante konzentrieren. Danach sei die Husserl'sche Phänomenologie naiv und unzureichend, da sie einer Metaphysik der Gegenwärtigkeit verhaftet bleibe. Zwar sei Husserl für seine Ausrichtung auf verschiedene Weisen der Gegebenheit zu loben, bedauerlicherweise aber lasse er „die Gegebenheit, deren Erweiterung er doch vollzogen habe, unbefragt ${ }^{\text {" }} .{ }^{26}$ Mit anderen Worten, Husserl versäume es, die grundlegende Frage nach der Gegebenheit als solcher zu stellen. Was heißt Geben, was steht mit der Tatsache, dass etwas gegeben sei, überhaupt auf dem Spiel? Dieses Versäumnis habe weitreichende Konsequenzen. Statt einer Orientierung an der Entsprechung zu den Dingen sei Husserl in seinen Untersuchungen von traditionellen, genauer, von cartesianischen Voraussetzungen und Entscheidungen geleitet. Deshalb bleibe Husserls Phänomenologie letztlich unphänomenologisch ${ }^{27}$ oder sei nur teilweise phänomenologisch. ${ }^{28}$ Die Phänomenalität der Phänomene werde auf die Gewissheit ihrer tatsächlichen Gegenwärtigkeit, ${ }^{29}$ auf als gesicherte Ständigkeit verstandene

25 Ebd., S. 245. Hervorhebung von mir.

26 J.-L. Marion, Réduction et donation. Recherches sur Husserl, Heidegger et la phénoménologie, Paris: PUF 1989, S. 62.

27 Ebd., S. 78.

28 Ebd., S. 124

29 Ebd., S. 81. 
Objektivität reduziert. Diese Fokussierung auf den objektiven Bestand gehe Hand in Hand mit Husserls Unvermögen, das Nicht-Gegenwärtige und Abwesende in den Blick zu nehmen. ${ }^{30}$ Deshalb sei das Husserl'sche Phänomen - als Gegenwärtigkeit für das Bewusstsein definiert und darauf beschränkt - ein flaches Phänomen, ein Phänomen ohne jegliche Tiefe. ${ }^{31}$ Doch genüge es nicht, einfach nur die Phänomene zu thematisieren - was Husserl ganz gut gelungen ist -, sondern wir müssen auch ihre Phänomenalität thematisieren. ${ }^{32}$ Ein solcher Schritt wird uns von einer Oberflächenphänomenologie zu einer Tiefenphänomenologie bringen. Dieser als der zweite phänomenologische Durchbruch (nach Husserls Logischen Untersuchungen) zu verstehenden Schritt wurde von Heidegger mit seiner Forderung nach einer Untersuchung des Seins von Seiendem vollzogen..$^{33}$ Eine andere Beschreibung von Husserls grundsätzlichem Fehler sei somit zu behaupten, er habe niemals verstanden, dass es das eigentliche Ziel der Phänomenologie sei, der Ontologie eine geeignete Methode zur Verfügung zu stellen. ${ }^{34}$ Natürlich könne man zu Husserls Verteidigung einwenden, und das ist so auch geschehen, dass er in Werken wie Cartesianische Meditationen, Ideen III, Erste Philosophie II und Formale und Transzendentale Logik in der Tat wiederholt von der ontologischen Dimension der Phänomenologie spreche. Doch die Entgegnung dürfte auf der Hand liegen: Selbst wenn Husserl früher noch als Heidegger darauf bestand, dass die Phänomenologie ontologische Implikationen habe, bedeutet das keineswegs, dass er unter Ontologie dasselbe versteht wie Heidegger. ${ }^{35}$ In Wirklichkeit sei Husserls Ontologie eine Ontologie von Objekten und bleibe es auch. Seiendes sei allein in dem Maße, wie ein es bestimmendes Urteil Bestand hat. So verfolge zum Beispiel seine Untersuchung der formalen Ontologie das Ziel, das allgemeine Substrat der Prädikation zu benennen. ${ }^{36}$ Doch das ist auch genau der Grund, warum die Husserl'sche Ontologie nicht zu einem Verständnis der von Heidegger aufgeworfenen Seinsfrage gelangen kann. Trotz seiner vielmaligen Hervorhebung des grundlegenden Unterschiedes zwischen Wirklichkeit und Bewusstsein habe Husserl niemals wirklich verstanden, dass dieser Unterschied auf einen ontologischen Unterschied, einen Unterschied im Sein hinauslaufe; vielmehr habe er Bewusstsein beständig als eine objek-

30 Ebd., S. 89.

31 Ebd., S. 90, S. 93, S. 97

32 Ebd., S. 99.

33 Ebd., S. 159.

34 Ebd., S. 213-214.

35 Ebd., S. 217.

36 Ebd., S. 230. 
tiv bestimmbare Region aufgefasst und es versäumt zu erkennen, dass dessen Seinsweise sich von der Seinsweise weltlicher Entitäten radikal unterscheide. ${ }^{37}$ Das heißt zusammengefasst: Husserls transzendentale Reduktion

„entspricht einer Konstitution von Objekten. (a) Sie entfaltet sich für das intentionale und konstituierende Ich. (b) Sie gibt ihm konstituierte Objekte, (c) erfasst in formalen Ontologien, die durch die formale Ontologie hindurch sämtlich dem Horizont der Gegenständlichkeit gemäß sind. (d) Sie schließt so aus der Gegebenheit alles das aus, was sich nicht auf die Gegenständlichkeit zurückführen lässt, nämlich die prinzipiellen Unterschiede von Seinsweisen (Bewusstsein, Zeughaftigkeit, Welt). ${ }^{438}$

Ich möchte mich auf einige Hauptkritikpunkte konzentrieren: 1) Die Husserl'sche Phänomenologie thematisiere nicht die eigentliche Phänomenalität der Phänomene. 2) Sie privilegiere ein aktives konstituierendes Ich. 3) Sie werde der spezifischen Seinsweise des Bewusstseins nicht gerecht, da sie dessen Sein beständig als eine Form von Objektsein interpretiere. 4) Letztlich habe die Husserl'sche Phänomenologie aufgrund ihrer Hervorhebung des objektiven Bestehens nur ein Auge für die Gegenwärtigkeit und versäume es, die Gegebenheit von Abwesendem und Nicht-Gegenwärtigem in Betracht zu ziehen.

Sollte sich diese Kritik als richtig erweisen, wäre es nur rechtens zuzugeben, dass die Husserl'sche Phänomenologie ernstzunehmenden Beschränkungen unterliegt, und dass der nach-Husserl'schen Phänomenologie nichts anderes übrig blieb als aus einem solchen Gerüst auszubrechen. Doch ist die Kritik berechtigt? Ich denke, nicht. Meines Erachtens hat die Husserl'sche Phänomenologie die Mittel, alle vier Einwände zu entkräften: Sie hat die eigentliche Dimension der Phänomenalität und nicht nur verschiedene Arten von Phänomenen untersucht. Sie hat wiederholt betont, in welchem Maße intentionale Aktivität Passivität voraussetzt. Sie hat die nicht-objektivierende Seinsweise des Bewusstseins analysiert, und sie hat das Wechselspiel zwischen Anwesenheit und Abwesenheit in extenso erörtert. Anders gesagt, ich denke, dass Husserl selbst ganz offensichtlich weit über das hinausging, was man eine flache Oberflächenphänomenologie nennen könnte. An Tiefe fehlt es nicht der Husserl'schen Phänomenologie, sondern der gängigen Kritik an ihr.

Man kann meines Erachtens ohne Umschweife zugestehen, dass zwischen einer Untersuchung, die beispielsweise den Unterschied zwischen Wahrnehmung, Einfühlung, Phantasie und Bildbewusstsein erkundet,

37 Ebd., S. 77, S. 187 f.

38 Ebd., S. 304. 
und der von Heidegger aufgeworfenen Seinsfrage ein wichtiger und entscheidender Unterschied besteht; jedoch erscheint es mir schlichtweg unehrlich, in Husserls Schriften über formale Ontologie nach einer möglichen Parallele für diese Radikalisierung zu suchen. Der offensichtliche und natürliche Ort dafür sind selbstverständlich vielmehr Husserls Schriften über die Zeit. Wie allgemein bekannt beschränkte sich Husserl in den Ideen I selbst auf eine Analyse der Beziehung zwischen konstituierten Objekten und konstituierendem Bewusstsein. Es ging ihm dabei um die Art und Weise, wie die Gegebenheit von Objekten durch Subjektivität bedingt ist; doch abgesehen von der Betonung, dass Erlebnisse nicht in derselben (perspektivischen) Weise gegeben sind wie Objekte, verfolgte er die Frage mit Bezug auf die Gegebenheit von Subjektivität selbst nicht weiter. Allerdings war sich Husserl durchaus bewusst, dass ein solches Schweigen phänomenologisch nicht hinnehmbar ist, und so gestand er auch unumwunden ein, dabei die wichtigsten und grundsätzlichsten Probleme, nämlich die das Zeitbewusstsein betreffenden, ausgelassen zu haben. ${ }^{39}$ Anders gesagt, jeder ernsthafte Versuch, die Radikalität des Unternehmens von Husserl zu beurteilen, muss notwendigerweise Husserls Schriften über die tiefsten Schichten der Konstitution behandeln. Er muss seine Schriften über Zeit und passive Synthesis behandeln. In diesen Schriften sind Husserls tiefgreifendste Reflexionen über die Struktur der Phänomenalität, die Natur der Subjektivität und das Wechselspiel von Anwesenheit und Abwesenheit zu finden. Soweit ich es übersehen kann, enthält Réduction et donation keinerlei Bezugnahme überhaupt auf eine dieser Schriften.

Ich kann aus Zeitgründen nicht näher auf Husserls subtile Analysen von Zeitbewusstsein, Selbstbewusstsein, Konstitution, Passivität, Faktizität, Alterität und Intersubjektivität eingehen. Es ist dies auch nicht notwendig, da in den letzten Jahren bereits all diese Bereiche von zahlreichen Husserl-Forschern ausgiebig erörtert wurden. ${ }^{40}$ Es liegt also ein

39 E. Husserl, Ideen zu einer reinen Phänomenologie und phänomenologischen Philosophie, S. 182.

40 Vgl. z.B. N. Lee, Edmund Husserls Phänomenologie der Instinkte, Dordrecht: Kluwer 1993; J. Benoist, Autour de Husserl, Paris: J. Vrin 1994; R. Bernet, La vie du sujet, Paris: PUF 1994; N. Depraz, Transcendance et incarnation. Le statut de l'intersubjectivité comme altérité à soi chez Husserl, Paris: J. Vrin 1995; A. Steinbock, Home and Beyond. Generative Phenomenology after Husserl, Evanston, IL: Northwestern University Press 1995; D. Zahavi, Husserl und die transzendentale Intersubjektivität. Eine Antwort auf die sprachpragmatische Kritik, Dordrecht: Kluwer 1996; N. Depraz, D. Zahavi (Hg.), Alterity and Facticity. New Perspectives on Husserl, Dordrecht: Kluwer 1998; D. Zahavi, Self-awareness and Alterity. A Phenomenological Investigation, Evanston, IL: Northwestern University Press 1999; A. Montavont, De la passivité dans la phénoménologie de 
umfängliches Korpus an Forschung vor, der von jeder ernsthaften Kritik an Husserl in Betracht gezogen werden muss. Stattdessen möchte ich, bevor ich zu meinem Hauptthema zurückkehre, kurz und bündig einfach nur ein paar Schlüsselpassagen aus verschiedenen Werken Husserls vorlegen, die direkt gegen einen der wiederholt geäußerten Kritikpunkte sprechen, nämlich dass Husserl das Sein von Subjektivität stets als eine Form von Objektsein betrachte.

Bereits in den Logischen Untersuchungen widerspricht Husserl jener Vorstellung. In der V. Logischen Untersuchung zum Beispiel schreibt Husserl, dass die intentionalen Erlebnisse erlebt werden, aber er verneint, dass sie gegenständlich erscheinen; sie werden nicht gesehen, nicht gehört. Sie sind bewusst, ohne dass sie intentionale Gegenstände sind. ${ }^{41}$ Es bleibt unbestritten, dass wir in der Tat unsere Aufmerksamkeit auf unsere Erlebnisse richten und sie dadurch zu Gegenständen einer inneren Wahrnehmung machen können, ${ }^{42}$ aber dies geschieht erst im Moment unserer Reflexion darauf. Im Gegensatz zu Brentano, der bekanntlich davon ausging, dass unsere Erlebnisse bewusst sind, indem sie als sekundäre Gegenstände genommen werden, will Husserl die (Selbst-)Gegebenheit von Erlebnissen ausdrücklich nicht mit der Gegebenheit von Objekten gleichsetzen. So schreibt er in der VI. Logischen Untersuchung: „Erlebtsein ist nicht Gegenständlichsein ", ${ }^{33}$ und 17 Jahre später in den Bernauer Manuskripten: „Sein Sein ist aber ein total anderes als das aller Objekte. Es ist eben Subjektsein [... “". ${ }^{44}$

In seiner Vorlesung Einleitung in die Logik und Erkenntnistheorie von 1906/07 finden wir Husserl mit einer ähnlichen Frage befasst. Er beginnt mit der Beobachtung, dass wir des Wahrnehmungsobjekts bewusst sind, wenn wir uns im Vorgang einer Wahrnehmung befinden. Aber wie steht es mit den Empfindungen und dem Wahrnehmungserlebnis selbst? Sie sind auch bewusst, aber nicht als Objekte der Wahrnehmung gegeben;

Husserl, Paris: PUF 1999; D. Zahavi, „Merleau-Ponty on Husserl. A Reappraisal“, in: L. Embree, T. Toadvine (Hg.), Merleau-Ponty's Reading of Husserl, Dordrecht: Kluwer Academic Publishers 2002, S. 3-29; D. Zahavi, Husserl's Phenomenology, Stanford: Stanford University Press 2003; D. Welton (Hg.), The New Husserl: A Critical Reader, Bloomington: Indiana University Press 2003; D. Zahavi, „Husserl's noema and the internalism-externalism debate", in: Inquiry 47/1 (2004), S. 42-66.

41 E. Husserl, Logische Untersuchungen. Zweiter Teil. Untersuchungen zur Phänomenologie und Theorie der Erkenntnis, S. 395, S. 399.

42 Ebd., S. 424.

43 Ebd., S. 669.

44 E. Husserl, Die Bernauer Manuskripte über das Zeitbewusstsein (1917/18) [Husserliana, Bd. XXXIII], hg. von R. Bernet und D. Lohmar, Dordrecht: Kluwer Academic Publishers 2001, S. 287. 
sie sind nicht wahrgenommen. ${ }^{45}$ Wir wissen, dass wir unsere Aufmerksamkeit vom wahrgenommenen Objekt ab- und dem Wahrnehmungserlebnis selbst zuwenden können. In diesem Sinne ist es möglich, auf das Erlebnis zu reflektieren. Um die Frage zu wiederholen, wie ist das Wahrnehmungserlebnis vor der Reflexion gegeben, wie ist es präreflexiv präsent? ${ }^{46}$ 1906/07 beantwortete Husserl die Frage mit der Unterscheidung zwischen Bewusstsein im Sinne von Erlebtsein und Bewusstsein im Sinne von Intentionalität. Während letzteres das Gerichtetsein auf ein Objekt impliziert, ist dies bei ersterem nicht der Fall. Wie Husserl ausdrücklich schreibt: „... ,erleben' bedeutet dann nicht ein Gegenständlich-Haben und auf das Gegenständliche in dieser oder jener Weise ,Beziehung haben ““. ${ }^{47}$ Und einige Seiten später fortführt:

„Nicht verwechseln darf man das Bewußtsein vom gegenständlichen Hintergrund und das Bewußtsein im Sinn des Erlebtseins. Erlebnisse als solche haben ihr Sein, aber sie sind nicht Gegenstände von Apperzeptionen (wir kämen ja sonst auf einen unendlichen Regreß). Der Hintergrund aber ist uns gegenständlich, er ist es durch den Komplex von apperzeptiven Erlebnissen, die ihn gleichsam konstituieren. Diese Gegenstände sind unbeachtet [...], aber etwas ganz anderes für uns als die bloßen Erlebnisse, z.B. die sie objektivierenden Apperzeptionen und Akterlebnisse selbst. [...] Das attentionale Bewußtsein des Hintergrunds und das Bewußtsein als bloßes Erlebtsein ist ganz zu scheiden. “48

Husserls Position war demnach relativ unmissverständlich. Ein intentionales Erlebnis ist einer Sache bewusst, die von ihm selbst verschieden ist, nämlich dem intendierten Gegenstand. Zugleich manifestiert das Erlebnis auch sich selbst. So wird das Erlebnis, außer dass es intentional ist, auch durch etwas charakterisiert, das Husserl gelegentlich sein „Urbewusstsein “49 nannte. Dieser Begriff Urbewusstsein, den Husserl bereits in der Vorlesung von 1906/07 verwandte, soll kein besonderes intentionales Erlebnis bedeuten. Vielmehr bezeichnet der Ausdruck die umfassende Dimension präreflexiven und nicht-objektivierenden Selbstbewusstseins, das ein wesentlicher Bestandteil jedes auftretenden Erlebnisses ist. ${ }^{50}$

45 E. Husserl, Einleitung in die Logik und Erkenntnistheorie [Husserliana, Bd. XXIV], hg. von U. Melle, Den Haag: M. Nijhoff 1984, S. 243.

46 Ebd., S. 244

47 Ebd., S. 247.

48 Ebd., S. 252.

49 E. Husserl, Zur Phänomenologie des inneren Zeitbewußteins (1893-1917) [Husserliana, Bd. X], hg. von R. Boehm, Den Haag: M. Nijhoff 1969, S. 89, S. 118-120.

50 E. Husserl, Einleitung in die Logik und Erkenntnistheorie, S. 245-247. 
Ich möchte ein letztes Mal zu den Bernauer Manuskripten zurückkehren, da sie Passagen enthalten, die eindeutig beweisen, dass Husserl nicht gewillt ist, die Seinsweise der Subjektivität umstandslos mit Objektsein gleichzusetzen. So schreibt er in einer recht bekannten Passage:

„In diesem Sinn ist es also nicht ,Seiendes', sondern Gegenstück für alles Seiende, nicht ein Gegenstand, sondern Urstand für alle Gegenständlichkeit. Das Ich sollte eigentlich nicht das Ich heißen, und überhaupt nicht heißen, da es dann schon gegenständlich geworden ist. Es ist das Namenlose über allem Fassbaren, < das > über allem nicht Stehende, nicht Schwebende, nicht Seiende, sondern ,Fungierende', als fassend, als wertend usw. " ${ }^{\text {11 }}$

Man könnte viel mehr darüber sagen, aber ich denke, es sollte auch so bereits deutlich geworden sein, dass ich die Heidegger'sche Kritik an Husserl für unbefriedigend halte. Marion weist natürlich zu Recht darauf hin, dass Husserl häufig über Heidegger zu uns gelangt. Doch gibt es irgendeinen Grund, der Heidegger'schen Husserl-Deutung einen bevorzugten Status einzuräumen? Gibt es irgendeinen Grund, Heideggers Darstellung der Beschränkungen der Husserl'schen Phänomenologie für eine besondere vertrauenswürdige Quelle zu halten? Ich denke, nicht. Nicht nur wegen ihrer begrenzten Textgrundlage - Heidegger bezieht sich im Großen und Ganzen lediglich auf die Logischen Untersuchungen und die Ideen I-, sondern vor allem, weil Heidegger seine eigenen Ziele verfolgte, seine eigenen Gründe hatte, seinem alten Lehrer gegenüber seine eigene Originalität unter Beweis stellen zu wollen.

In seiner kürzlichen Auseinandersetzung mit dem Werk des Neukantianers Emil Lask legte Steven Crowell dar, wie Lask den Unterschied zwischen einer positiven (empirischen oder metaphysischen) Untersuchung und einer transzendentalen Untersuchung als Unterschied zwischen einer direkten Thematisierung von Entitäten mit dem Ziel der Offenlegung ihrer materiellen Eigenschaften und einer reflexiven Thematisierung der Wahrheitsstruktur oder Intelligibilität von Entitäten geltend machte. Lask arbeitet demnach mit einem Dualismus zwischen dem Reich der (physischen oder metaphysischen) Entitäten auf der einen Seite und dem Reich der Geltung (Wahrheit, Intelligibilität) auf der anderen Seite. Dieser Unterschied zwischen dem, was ist, und dem, was gilt, ist im Grunde eine Unterscheidung zwischen der Entität und ihrem Sinn, was wiederum kein empirischer, sondern vielmehr ein transzendentaler Unterschied ist zwischen der Art und Weise, wie ein und diesel-

51 E. Husserl, Die Bernauer Manuskripte über das Zeitbewußtsein (1917/18), S. 277 f. 
be Entität zunächst einmal in direkter Erfahrung und dann wiederum in reflexiver Analyse aufgenommen werden kann. Lask ließ somit keinerlei Zweifel am reflexiven Charakter der Transzendentalphilosophie, deren erstes Prinzip nicht eine Entität, Subjekt oder Substanz, sondern etwas Grundlegenderes ist, nämlich Sinn. ${ }^{52}$

Lasks Sichtweise wird in weitem Maße von Husserl geteilt, mit dem einen Unterschied, dass für Husserl - und das ist ein wichtiger Bereich, in dem die Phänomenologie vom Neukantianismus abweicht - die Erforschung von Sinn auf eine Theorie der Intentionalität gegründet sein muss, da allein eine solche Theorie uns Klarheit hinsichtlich Evidenz und Gegebenheit verschaffen kann, wie wir sie zur Vermeidung jeglichen Dogmatismus benötigen. So war Husserl im Gegensatz zu Lask nicht der Meinung, dass eine transzendentale Sinntheorie ohne eine gleichzeitige Analyse der subjektiven Dimension der Sinnerschließung durchgeführt werden kann. ${ }^{53}$

Ist es möglich, die Rolle von Subjektivität in der Erschließung von Sinn hervorzuheben, ohne dabei gleichzeitig das Subjekt zur ausschließlichen Instanz zu erklären, die die Bildung von Sinn bestimmt und kontrolliert? Zweifellos ist das möglich, denn es besteht ein entscheidender Unterschied, ob man das Subjekt als notwendige oder ob man es als hinreichende Bedingung der Möglichkeit des Sichzeigens ausweist. Mit anderen Worten, es ist sehr wohl möglich, Husserls Untersuchung der konstituierenden Subjektivität als eine Untersuchung des Dativs des Sichzeigens zu lesen. Es ist meines Erachtens kein Zufall, dass Husserl sehr oft die Formulierung gebraucht: „Etwas konstituiert sich im Bewusstsein.“

Auf jeden Fall und entgegen einer weit verbreiteten Fehldeutung hat Husserl in seiner Darstellung der Konstitution das Problem der Passivität nicht übersehen, sondern dieser wichtigen Frage in Wirklichkeit zahlreiche Analysen gewidmet. Auch wenn unser Ausgangspunkt in Akten besteht, in denen das Subjekt aktiv Position bezieht, Akten, in denen das Subjekt vergleicht, unterscheidet, urteilt, etwas wünscht oder will, ist Husserl doch sogleich bereit darzulegen, dass das Subjekt, wann immer es aktiv ist, auch passiv ist, da aktiv zu sein auf etwas zu reagieren heißt. ${ }^{54}$ So schreibt Husserl:

52 S. G. Crowell, Husserl, Heidegger, and the Space of Meaning, Evanston: Northwestern University Press 2001, S. 45, S. 51, S. 89.

53 Ebd., S. 54, S. 58.

54 E. Husserl, Ideen zu einer reinen Phänomenologie und phänomenologischen Philosophie. Zweites Buch: Phänomenologische Untersuchungen zur Konstitution, S. 213, S. 337. 
„[I]chliche Aktivität setzt Passivität voraus - ichliche Passivität - und beides setzt voraus Assoziation und Vorbewusstsein in Form des letztlich hyletischen Untergrundes. “55

„Die Strukturanalyse der urtümlichen Gegenwart (das stehend lebendige Strömen) führt uns auf die Ichstruktur und die sie fundierende ständige Unterschichte des ichlosen Strömens, das durch eine konsequente Rückfrage auf das, was auch die sedimentierte Aktivität möglich macht und voraussetzt, auf das radikal Vor-Ichliche zurückleitet. ${ }^{\text {" }} 6$

„[...]das Strömen geschieht, der Strom ist nicht aus einem Tun des Ich, als ob es darauf gerichtet wäre, es zu verwirklichen, als ob es sich verwirklichte aus einem Tun. Es ist also kein Getanes, keine Tat (im weitesten Sinne). "

Husserl gestand folgerichtig zu, dass im Untergrund, in der Tiefendimension von Subjektivität anonyme und nicht willentlich beeinflussbare Konstitutionsprozesse stattfinden. ${ }^{58}$

\section{Schlussfolgerung}

Dieser Versuch einer Verteidigung Husserls ist nicht so aufzufassen, als impliziere er, es gäbe keinen Raum für eine Verbesserung. Den gibt es natürlich. Ich gestehe gerne zu, dass Husserl in diesen Fragen nicht immer so eindeutig war, wie man es sich wünschen möchte. Man sollte zwischen Husserls Phänomenologie und der Husserl'schen Phänomenologie unterscheiden. Es ist durchaus möglich, erstere durch letztere zu kritisieren. Kurz gesagt, es geht nicht darum, ob Husserls Phänomenologie Mängel hat, die es zu verbessern gilt, sondern vielmehr darum, ob die Husserl'sche Phänomenologie konstitutionelle Schwächen hat, die sie für eine Begründung der Phänomenologie entsprechend den Anforderungen unserer Zeit ungeeignet machen. Wie inzwischen deutlich geworden sein sollte, lautet meine Antwort, dass ungeachtet aller Verdienste oder möglicher Schwächen seiner konkreten phänomenologischen Analysen die

55 E. Husserl, Ms. C 3 41b-42a. Ich danke dem Direktor des Husserl-Archivs in Leuven, Prof. Dr. Rudolf Bernet, für die Erlaubnis zur Einsichtnahme in Husserls unveröffentlichte Manuskripte.

56 E. Husserl, Zur Phänomenologie der Intersubjektivität. Texte aus dem Nachlass. Dritter Teil. 1929-35, S. 598. - Hervorhebung von mir.

57 E. Husserl, Zur phänomenologischen Reduktion. Texte aus dem Nachlass (1926-1935), S. 179.

58 E. Husserl, Phänomenologische Psychologie. Vorlesungen Sommersemester 1925 [Husserliana, Bd. IX], hg. von W. Biemel, Den Haag: M. Nijhoff 1968, S. 514; ders., Ideen zu einer reinen Phänomenologie und phänomenologischen Philosophie. Zweites Buch: Phänomenologische Untersuchungen zur Konstitution, S. $276 \mathrm{f}$. 
Husserl'sche Methodologie Kernstücke enthält, die für die gegenwärtige Phänomenologie unentbehrlich bleiben. Warum hat Husserl darauf bestanden, dass wir die Reduktion vollziehen müssen, wenn wir Phänomenologie betreiben wollen? Soweit ich sehe, besteht das letzte Ziel des Verfahrens der Reduktion nicht darin, Gegenstände oder Erlebnisse so präzise und akribisch wie möglich zu beschreiben, und die Reduktion zielt auch nicht auf eine erschöpfende Erkundung der Phänomene in ihrer ganzen faktischen Verschiedenartigkeit ab. Nein, ihre wahre Aufgabe ist es, die Phänomene als Phänomene zu untersuchen; es geht ihr also um das Verstehen der Dimension von Phänomenalität überhaupt, um die Erforschung ihrer innersten Struktur und der eigentlichen Bedingung ihrer Möglichkeit. Diese Aufgabe ist eine transzendentalphilosophische. Es handelt sich um einen Schritt von einer direkten metaphysischen oder empirischen Untersuchung von Gegenständen zu einer Untersuchung des Gerüsts an Sinn und Intelligibilität, das jede solche direkte Untersuchung überhaupt erst ermöglicht. Eine solche Untersuchung des Wesens der Manifestation - um einen Terminus von Michel Henry zu verwenden - ist fundamentaler und früher als jede Spaltung zwischen psychischer Innerlichkeit und physischer Äußerlichkeit, da es sich um eine Untersuchung der Dimension handelt, in der jegliches Objekt, sei es intern oder extern, sich manifestiert.

Um es noch einmal zu sagen, meines Erachtens ist der Begriff der Reduktion der ursprüngliche Durchbruch. Er ist der reflexive Schritt, der das Feld phänomenologischer Forschung ein für alle Mal eröffnet hat. Es ist eine Eröffnung, die in jeder vorgeschlagenen Radikalisierung nachfolgender Phänomenologen vorausgesetzt wird. Mehr noch, entgegen weit verbreiteten Missverständnissen ist dieser reflexive Schritt per se weder einem cartesianischen Internalismus, einer naiven Metaphysik der Gegenwärtigkeit noch einer Privilegierung eines aktiven, die Geschehnisse restlos kontrollierenden Ich verpflichtet.

Mit dieser Klarstellung im Sinn halten wir es für möglich, ganz kurz zu der eingangs gestellten Frage zurückzukehren: Warum wurden die phänomenologischen Reflexionen über die Erzeugung von Sinn zum Beispiel von den empirischen Untersuchungen nicht-bewusster Informationsverarbeitung im eigentlichen Sinne nicht vorweggenommen (und vielleicht sogar ersetzt)? Eine mögliche Antwort wäre, dass die die nichtbewusste Informationsverarbeitung betreffenden Ergebnisse Resultate einer aus der Dritte-Person-Perspektive durchgeführten Untersuchung sind. Dies unterscheidet sie grundsätzlich von der phänomenologischen Untersuchung, die der Erste-Person-Perspektive und der subjektiven Dimension von Sinnerschließung gerecht zu werden versucht. Das Pro- 
blematische dieser Antwort besteht darin, dass sie ausreichend und angemessen erscheint, wenn es sich um gewöhnliche phänomenologische Analysen handelt, dass sie aber weniger auf eine Untersuchung der Tiefendimension passt, wie sie derzeit im Zentrum des Interesses steht. Deshalb haben wahrscheinlich auch einige wie beispielsweise Janicaud in Frage stellen wollen, ob eine solche Tiefenuntersuchung tatsächlich überhaupt noch phänomenologisch genannt werden kann. Aber vielleicht ist auch eine andere Antwort möglich. Während die phänomenologische Untersuchung - in einem weiten Sinne des Wortes - eine transzendentale ist, da sie die wesentlichen Bedingungen der Erzeugung von Sinn zu untersuchen und zu analysieren sucht und darin auch die Bedingungen einschließt, die bei einer wissenschaftlichen Untersuchung in dritter Person vorausgesetzt werden, legt die psychologische Untersuchung einen eher objektivistischen Zugang zum Sinn zugrunde. Sinn scheint für sie etwas zu sein, das einfach da ist - fertig vorhanden -, und damit bleibt allein die Frage, wie Sinn vom Gehirn entdeckt und verarbeitet wird. Wie auch immer man ein solches Unternehmen beurteilen mag, es ist mit Sicherheit keine erschöpfende Behandlung des Problems.

Ich denke, beide Erwiderungen fangen etwas Wesentliches am phänomenologischen Unternehmen ein. Ob sie uns auch ein für alle Mal in die Lage versetzen, zwischen empirischer Psychologie und Kognitionswissenschaft einerseits und Phänomenologie andererseits zu unterscheiden, ist eine andere Frage. Ich fürchte, dass wir es uns mit einer sehr klar geschnittenen Abgrenzung zu einfach machen. Aber dies wäre das Thema für einen weiteren Aufsatz. ${ }^{59}$ Abschließend möchte ich nur bemerken, dass jeder Phänomenologe unserer Zeit, der Husserls Ausrichtung auf die Erste-Person-Perspektive als zu cartesianisch und die auf das Problem der Transzendentalität gelegte Betonung als zu kantianisch zurückweist, der aber gleichwohl geltend machen möchte, dass seine Betonung der anonymen Natur des Sinnereignisses sich von den Behauptungen naturalistischer, strukturalistischer oder spekulativ metaphysischer Theorien (um nur einige wenige Kandidaten zu nennen) wesentlich unterscheidet, uns nicht nur eine Erklärung schuldet, worin jener Unterschied bestehen soll, sondern auch eine Erklärung, warum sein eigenes Unternehmen die Bezeichnung "phänomenologisch" verdient. ${ }^{60}$

Übersetzt aus dem Englischen von Hans-Dieter Gondek

59 Vgl. D. Zahavi, „Phenomenology and the Project of Naturalization“, in: Phenomenology and the Cognitive Sciences 3/4 (2004), S. 331-347.

60 Diese Studie wurde von der Nationalen Forschungsstiftung Dänemarks ermöglicht. 